\title{
Positronium-hydrogen-atom scattering in a five-state model
}

\author{
Sadhan K. Adhikari and P. K. Biswas \\ Instituto de Física Teórica, Universidade Estadual Paulista 01 405-900 São Paulo, São Paulo, Brazil
}

(Received 1 October 1998; revised manuscript received 1 February 1999)

\begin{abstract}
The scattering of orthopositronium (Ps) by hydrogen atoms has been investigated in a five-state coupledchannel model allowing for $\mathrm{Ps}(1 s) \mathrm{H}(2 s, 2 p)$ and $\mathrm{Ps}(2 s, 2 p) \mathrm{H}(1 s)$ excitations using a recently proposed electron-exchange model potential. The higher $(n \geqslant 3)$ excitations and ionization of the Ps atom are calculated using the first Born approximation. Calculations are reported of scattering lengths, phase shifts, elastic, Ps and $\mathrm{H}$ excitation, and total cross sections. Remarkable correlations are observed between the $S$-wave Ps-H binding energy and the singlet scattering length, effective range, and resonance energy obtained in various model calculations. These correlations suggest that if a Ps-H dynamical model yields the correct result for one of these four observables, it is expected to lead to the correct result for the other three. The present model, which is constructed so as to reproduce the Ps-H resonance at $4.01 \mathrm{eV}$, automatically yields a Ps-H bound state at $-1.05 \mathrm{eV}$ that compares well with the accurate value of $-1.067 \mathrm{eV}$. The model leads to a singlet scattering length of $3.72 a_{0}$ and effective range of $1.67 a_{0}$, whereas the correlations suggest the precise values of $3.50 a_{0}$ and $1.65 a_{0}$ for these observables, respectively. [S1050-2947(99)07703-3]
\end{abstract}

PACS number(s): 34.10.+x, 36.10.Dr

\section{INTRODUCTION}

Recently, there have been several experimental and theoretical investigations of orthopositronium (Ps) atom scattering from different neutral atomic and molecular targets. Experiments have primarily measured total Ps-atom scattering cross sections from various targets [1-3]. In addition to total cross sections, the theoretical studies have also predicted partial cross sections and phase shifts for Ps-H [4-9], Ps-He $[8,10-12]$, and Ps- $\mathrm{H}_{2}[13,14]$ systems. Ps scattering by neutral targets is of special interest, as the direct amplitudes for elastic and even-parity state transitions are zero [15] due to the internal charge and mass symmetry of Ps. Hence the electron-exchange interaction is the dominating factor at low energies in any Ps-impact scattering with neutral targets apart from the effect of polarization and van der Waals forces $[10,16]$. Ps scattering makes it possible to study the effect of exchange in an environment characteristically different from that of the electron-atom systems [15]. Among all Ps-atom systems, the positronium-hydrogen (Ps-H) system is the simplest and is of fundamental interest. The Ps-H scattering has most of the complications of a many-body problem, but few-body techniques can be employed for its solution.

A general feature of the measured total cross section in Ref. [1] for Ps scattered by $\mathrm{He}, \mathrm{Ar}$, and $\mathrm{H}_{2}$, is a peak near 20-25 eV and a decreasing trend below this energy. Recent measurements near $1 \mathrm{eV}$ [2] are consistent with this trend. However, because of the large error bars on the measurement in Ref. [1] at the lowest energy $(10 \mathrm{eV})$ and due to inadequate data in this energy region, it is not clear from experiment whether the total cross section has a minimum near the Ps excitation threshold or not. The recent three-Ps-state studies of Refs. $[9,12,14]$ suggest the existence of a minimum near the Ps $(2 s)$ threshold. This feature of the cross section is able to reconcile the two different experimental findings and is also noticed in the unpublished theoretical work of Peach [17]. The $R$-matrix calculation $[7,8]$ for $\mathrm{H}$ and $\mathrm{He}$, in which
22 coupled pseudostates are included, does not show this trend; whereas the static-exchange (one-state) cross sections [10] for He are too large to match the measurement near the $\operatorname{Ps}(2 s)$ threshold [12]. In this respect, the model-potential studies of Refs. $[9,12,14]$ are unique in reproducing the experimental trend of Ps-impact scattering by $\mathrm{H}, \mathrm{He}$, and $\mathrm{H}_{2}$. Unphysically large low-energy cross sections of previous calculations are expected to be a consequence of the nonorthogonality arising from antisymmetrization coupled with inadequate correlations in the exchange dominated Ps scattering.

In this paper we present a theoretical study of ortho-Ps-H scattering employing a five-state model allowing for excitation of both Ps and $\mathrm{H}$ atoms using the model exchange potential mentioned above. The following states are included in the calculation: $\operatorname{Ps}(1 s) \mathrm{H}(1 s), \operatorname{Ps}(2 s) \mathrm{H}(1 s), \operatorname{Ps}(2 p) \mathrm{H}(1 s)$, $\mathrm{Ps}(1 s) \mathrm{H}(2 s)$, and $\mathrm{Ps}(1 s) \mathrm{H}(2 p)$ and such a model should be considered adequate at low energies. The cross sections for higher discrete and continuum excitations of the Ps atom are calculated in the framework of Born approximation. These Born cross sections are added to the above five-state cross sections to predict the total cross section.

The parametrization of the model exchange potential of Ref. [12] was obtained using a physical argument, and leaves an option for the parameters to be varied to tune to some precise data at low energies. In the absence of experimental Ps-H cross sections we tune this parameter to reproduce the energy of the singlet $S$-wave resonance. Ho has provided the most precise estimate of $S$-wave resonance energy, which is $4.01 \mathrm{eV}$ (width $0.075 \mathrm{eV}$ ) [18]. Frolov and Smith have made, to our knowledge, the most accurate estimate of the $S$-wave bound state, which is $1.067 \mathrm{eV}$ [19]. We varied the parameter of our model to fit the Ps-H resonance energy at $4.01 \mathrm{eV}$ and found that the same model without any further adjustment also produced a Ps-H bound state at $-1.05 \mathrm{eV}$. The present calculation has been able to produce these two features of the Ps-H system simultaneously and precisely. Similar to those obtained in the three-nucleon sys- 
tem [20,21], we find remarkable correlations between the $S$-wave Ps-H binding energy and the singlet scattering length, effective range, and resonance energy obtained in various model calculations. These correlations suggest that if a model yields the correct result for one of these observables it should also yield the correct result for the other three. The present model leads to reasonably accurate energies for the Ps-H bound state and resonance and the singlet scattering length and effective range are also expected to be fairly accurate, as can be seen from the correlations.

We describe the calculational scheme, model exchange potential and numerical results in Sec. II and a summary of our findings in Sec. III.

\section{MODEL POSITRONIUM-HYDROGEN CALCULATION}

\section{A. Calculational scheme}

The total antisymmetrized wave function for the Ps- $\mathrm{H}$ system allowing excitation of both $\mathrm{Ps}$ and $\mathrm{H}$ is given by

$$
\begin{aligned}
\Psi^{ \pm}\left(\mathbf{r}_{1}, \mathbf{r}_{2}, \mathbf{x}\right) \equiv & \frac{1}{\sqrt{2}} \sum_{\mu, \nu}\left[\phi_{\mu}\left(\mathbf{r}_{2}\right) \chi_{\nu}\left(\mathbf{t}_{1}\right) F_{\mu \nu}\left(\mathbf{s}_{1}\right)\right. \\
& \left. \pm \phi_{\mu}\left(\mathbf{r}_{1}\right) \chi_{\nu}\left(\mathbf{t}_{2}\right) F_{\mu \nu}\left(\mathbf{s}_{2}\right)\right]
\end{aligned}
$$

where $\mathbf{s}_{j}=\left(\mathbf{x}+\mathbf{r}_{j}\right) / 2$ and $\mathbf{t}_{j}=\left(\mathbf{x}-\mathbf{r}_{j}\right), j=1,2$, with $\mathbf{x}$ the positron coordinate and $\mathbf{r}_{j}$ the coordinates of the two electrons, $\phi_{\mu}\left(\chi_{\nu}\right)$ the bound-state wave function of $\mathrm{H}(\mathrm{Ps})$ in quantum state $\mu(\nu)$, and $F_{\mu \nu}$ the continuum orbital of Ps with respect to $\mathrm{H}$. The Schrödinger equation for this wave function when projected on the final $\mathrm{H}$ and Ps states $\phi_{\mu^{\prime}}$ and $\chi_{\nu^{\prime}}$, respectively, leads to the following Lippmann-Schwinger scattering integral equation in momentum space:

$$
\begin{aligned}
f_{\mu^{\prime} \nu^{\prime}, \mu \nu}^{ \pm}\left(\mathbf{k}^{\prime}, \mathbf{k}\right)= & \mathcal{B}_{\mu^{\prime} \nu^{\prime}, \mu \nu}^{ \pm}\left(\mathbf{k}^{\prime}, \mathbf{k}\right)-\sum_{\mu^{\prime \prime}, \nu^{\prime \prime}} \int \frac{\mathrm{d} \mathbf{k}^{\prime \prime}}{2 \pi^{2}} \\
& \times \frac{\mathcal{B}^{ \pm}{ }_{\mu^{\prime} \nu^{\prime}, \mu^{\prime \prime} \nu^{\prime \prime}}\left(\mathbf{k}^{\prime}, \mathbf{k}^{\prime \prime}\right) f_{\mu^{\prime \prime} \nu^{\prime \prime}, \mu \nu}^{ \pm}\left(\mathbf{k}^{\prime \prime}, \mathbf{k}\right)}{E-\mathcal{E}_{\mu^{\prime \prime}}-\epsilon_{\nu^{\prime \prime}}-k^{\prime \prime 2} / 4+\mathrm{i} 0},
\end{aligned}
$$

where the singlet and triplet "Born" amplitudes $B^{ \pm} \quad$ are given by $\mathcal{B}_{\mu^{\prime} \nu^{\prime}, \mu \nu}^{ \pm}\left(\mathbf{k}_{\mathbf{f}}, \mathbf{k}_{\mathbf{i}}\right)=g_{\mu^{\prime} \nu^{\prime}, \mu \nu}^{D}\left(\mathbf{k}_{\mathbf{f}}, \mathbf{k}_{\mathbf{i}}\right)$ $\pm g_{\mu^{\prime} \nu^{\prime}, \mu \nu}^{E}\left(\mathbf{k}_{\mathbf{f}}, \mathbf{k}_{\mathbf{i}}\right)$, where $g^{D}$ and $g^{E}$ represent the direct and exchange Born amplitudes and the $f^{ \pm}$are the singlet and triplet scattering amplitudes, respectively. The energies of the intermediate Ps and $\mathrm{H}$ states are $\epsilon_{\nu^{\prime \prime}}$ and $\mathcal{E}_{\mu^{\prime \prime}}$ and $E$ is the total energy of the system. The differential cross section is defined by

$$
\left(\frac{d \sigma}{d \Omega}\right)_{\mu^{\prime} \nu^{\prime}, \mu \nu}=\frac{k^{\prime}}{4 k}\left[\left|f_{\mu^{\prime} \nu^{\prime}, \mu \nu}^{+}\left(\mathbf{k}^{\prime}, \mathbf{k}\right)\right|^{2}+3\left|f_{\mu^{\prime} \nu^{\prime}, \mu \nu}^{-}\left(\mathbf{k}^{\prime}, \mathbf{k}\right)\right|^{2}\right],
$$

and the quenching cross section that describes conversion from orthopositronium to parapositronium is defined by

$$
\left(\frac{d \sigma}{d \Omega}\right)_{\mu^{\prime} \nu^{\prime}, \mu \nu}^{\text {quen }}=\frac{k^{\prime}}{16 k}\left|f_{\mu^{\prime} \nu^{\prime}, \mu \nu}^{+}\left(\mathbf{k}^{\prime}, \mathbf{k}\right)-f_{\mu^{\prime} \nu^{\prime}, \mu \nu}^{-}\left(\mathbf{k}^{\prime}, \mathbf{k}\right)\right|^{2} .
$$

\section{B. Model exchange potential}

The derivation of the model exchange potential has been adequately described recently and here we quote the principal results. The Ps-H model exchange potential is given by $[12,22]$

$$
\begin{aligned}
g_{\mu^{\prime} \nu^{\prime}, \mu \nu}^{E}\left(\mathbf{k}_{\mathbf{f}}, \mathbf{k}_{\mathbf{i}}\right)= & \frac{4(-1)^{l+l^{\prime}}}{D} \\
& \times \int \phi_{\mu^{\prime}}^{*}(\mathbf{r}) \exp (\mathrm{i} \mathbf{Q} \cdot \mathbf{r}) \phi_{\mu}(\mathbf{r}) \mathrm{d} \mathbf{r}_{2} \\
& \times \int \chi_{\nu^{\prime}}^{*}(\mathbf{t}) \exp (\mathrm{i} \mathbf{Q} \cdot \mathbf{t} / 2) \chi_{\nu}(\mathbf{t}) \mathrm{d} \mathbf{t},
\end{aligned}
$$

with

$$
D=k_{f}^{2} / 4+\alpha_{\mu}^{2}+\beta_{\nu^{\prime}}^{2},
$$

where $l$ and $l^{\prime}$ are the angular momenta of the initial and final Ps states, the initial and final Ps momenta are $\mathbf{k}_{\mathbf{i}}$ and $\mathbf{k}_{\mathbf{f}}, \mathbf{Q}=\mathbf{k}_{\mathbf{i}}-\mathbf{k}_{\mathbf{f}} \cdot \alpha_{\mu}^{2} / 2$ and $\alpha_{\mu^{\prime}}^{2} / 2$, and $\beta_{\nu}^{2}$ and $\beta_{\nu^{\prime}}^{2}$, are the binding energies of the initial and final states of $\mathrm{H}$ and Ps in atomic units $(\mathrm{au})$, respectively. The factor $(-1)^{l+l^{\prime}}$ provides the correct sign of the exchange potential given by formal antisymmetrization. The model exchange potential given by Eqs. (5) and (6) is not time-reversal symmetric. A timereversal symmetric form has also been suggested in which Eq. (6) is replaced by [12]:

$$
D=\left(k_{i}^{2}+k_{f}^{2}\right) / 8+\left(\alpha_{\mu}^{2}+\alpha_{\mu^{\prime}}^{2}\right) / 2+\left(\beta_{\nu}^{2}+\beta_{\nu^{\prime}}^{2}\right) / 2,
$$

which leaves the elastic Born results unchanged.

\section{Numerical results}

After a partial-wave projection, the singlet $(+)$ and triplet (-) scattering equations (2) are solved by the method of matrix inversion. The maximum number of partial waves included in the calculation is ten. Contribution of higher partial waves to cross sections is included by using the Born terms.

In our latest calculations [12] we find that the symmetric form provides better results and therefore here we present results of Ps-H scattering using a five-state model and Eqs. (5) and (7) that includes the following states: $\mathrm{Ps}(1 s) \mathrm{H}(1 s)$, $\mathrm{Ps}(2 s) \mathrm{H}(1 s), \quad \operatorname{Ps}(2 p) \mathrm{H}(1 s), \quad \operatorname{Ps}(1 s) \mathrm{H}(2 s), \quad$ and $\operatorname{Ps}(1 s) \mathrm{H}(2 p)$. The truncated model that includes the first three of these states will be referred to as the three-Ps-state, or simply, the three-state, model, and the three-H-state model includes the first, fourth, and fifth of this set. The model that includes the first $n$ states of this set will be termed the $n$-state model. The Born potentials for the simultaneous excitation of both $\mathrm{H}$ and Ps atoms are found to be very small and will not be considered here in the coupled-channel scheme. Higher excitations and ionization of Ps are conveniently treated in the Born approximation including exchange and 


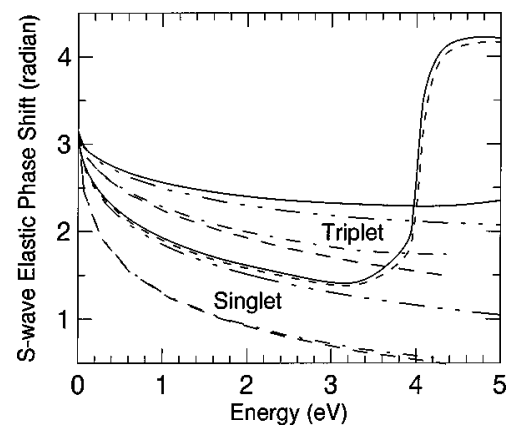

FIG. 1. $S$-wave elastic scattering phase shifts for singlet and triplet states at different Ps energies: present five-state (full line), present two-state (dotted line), present static exchange (dasheddouble-dotted line), Hara and Fraser (dashed line, Ref. [5]), Sinha, Chaudhury, and Ghosh (dashed-dotted line, Ref. [6]).

higher excitations and ionization of $\mathrm{H}$ are excluded. We calculate the elastic $\operatorname{Ps}(1 s) \mathrm{H}(1 s)$ cross section and inelastic exci-

tation cross sections to $\operatorname{Ps}(2 s) \mathrm{H}(1 s), \quad \operatorname{Ps}(2 p) \mathrm{H}(1 s)$, $\operatorname{Ps}(1 s) \mathrm{H}(2 s)$, and $\mathrm{Ps}(1 s) \mathrm{H}(2 p)$ states. We also calculate cross sections for the discrete excitation of the $3 s, 3 p, 3 d, 4 p, 4 d, 4 f, 5 p, 5 d, 5 f$, and $6 p$ states and also for ionization of Ps in the first Born approximation, keeping the target frozen to its initial ground state using the exchange given by Eqs. (5) and (7).

In previous studies we found that the exact values of the parameters $\alpha$ and $\beta$ in Eqs. (6) and (7) lead to good results for cross sections. However, these parameters in Eqs. (6) and (7) correspond to some average value of momentum [12] and it was noted that one could conveniently allow these parameters to vary in order to improve the fit with experiment. As there are no experiments in Ps-H scattering, we choose these parameters to fit the known $S$-wave singlet resonance at 4.01 $\mathrm{eV}$ in the Ps-H system [18]. The resonance is found in the two-state model with $\mathrm{Ps}(1 s) \mathrm{H}(1 s)$ and $\mathrm{Ps}(2 s) \mathrm{H}(1 s)$ states. It continues to exist as more states are included in the dynamical equation. However, its energy reduces a little (by up to approximately $0.05 \mathrm{eV}$ ) as more and more states are added. The position of the resonance in the five-state and

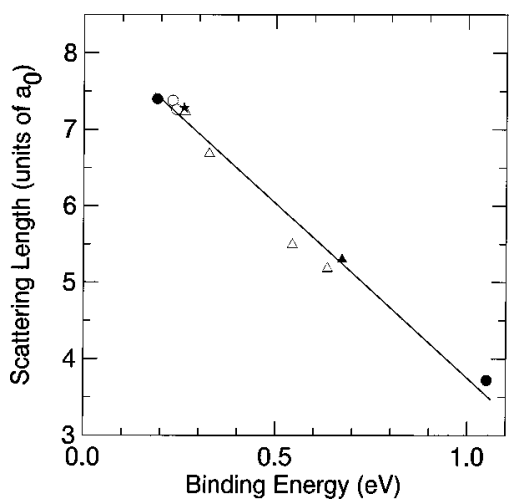

FIG. 2. The singlet scattering length vs binding energy of different models: open triangles (Ref. [7]), open circles (calculated from phase shifts of Ref. [6]), solid triangle (from Ref. [4]), star (as calculated in Ref. [4] from phase shifts of [5]), solid circle [fivestate calculation with $C=1$ and 0.784 in Eq. (8)], and full line (straight line fit).

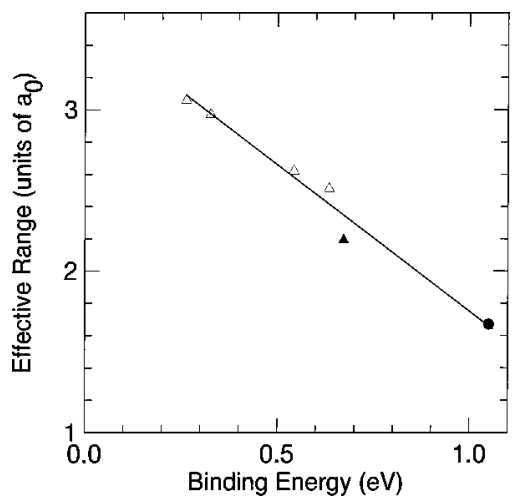

FIG. 3. $S$-wave singlet effective range vs binding energy of different models: open triangles (Ref. [7]), solid triangle (from Ref. [4]), solid circle [five-state calculation with $C=0.784$ in Eq. (8)], and full line (straight line fit).

two-state models with the exact parameters $\alpha$ 's and $\beta$ 's in Eq. (7) is $4.72 \mathrm{eV}$ and $4.76 \mathrm{eV}$, respectively. We find that the Ps-H resonace energy decreases and the binding energy increases monotonically, as the values of the parameters $\alpha$ 's and $\beta$ 's are reduced in Eq. (7). For a systematic reduction we used in place of Eq. (7) the following form:

$$
D=\left(k_{i}^{2}+k_{f}^{2}\right) / 8+C^{2}\left[\left(\alpha_{\mu}^{2}+\alpha_{\mu^{\prime}}^{2}\right) / 2+\left(\beta_{\nu}^{2}+\beta_{\nu^{\prime}}^{2}\right) / 2\right],
$$

where $C$ is an arbitrary factor. In order to obtain the $S$-wave resonance at $4.01 \mathrm{eV}$ in the five-state model we need $C$ $=0.784$, which is the most accurate estimate of this energy [18]. Interestingly enough, with this value of $C$, the five-state model produces a Ps- $\mathrm{H}$ bound state at $-1.05 \mathrm{eV}$, which is consistent with both the accurate theoretical estimate of $-1.067 \mathrm{eV}[19]$ and experimental result of $-1.1 \pm 0.2 \mathrm{eV}$ [23]. The binding energy is calculated by extrapolating the calculated $k \cot \delta$ values at positive energies to negative energies using the following effective-range expansion: $k \cot \delta=-1 / a+r_{0} k^{2} / 2+B k^{4}$, and finding the solution of $k \cot \delta-i k=0$ at the bound state, where $\delta$ is the $S$-wave phase shift, $a$ the scattering length, $r_{0}$ the effective range, $k$ the momentum, and $B$ the coefficient of the $k^{4}$ term. In all calculations presented in this work we use the value of $C$ $=0.784$ in Eqs. (8) and (5). The simultaneous accurate reproduction of both the binding and resonance energies assures the reliability of our model.

The elastic scattering $S$-wave phase shifts for different partial waves for singlet and triplet states below the lowest

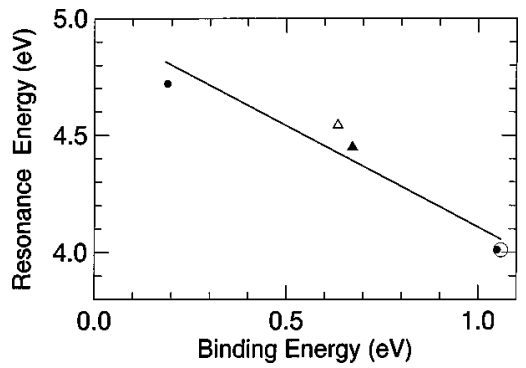

FIG. 4. $S$-wave singlet resonance vs binding energy of different models: open circle (Refs. [18,19]), open triangle (Ref. [7]), solid triangle (from Ref. [4]), solid circle [five-state calculation with $C$ $=1$ and 0.784 in Eq. (8)], and full line (straight line fit). 
TABLE I. Singlet scattering length and effective range in units of $a_{0}$ and $S$-wave singlet binding energies $\left(E_{B}\right)$ in $\mathrm{eV}$ for different numbers of coupled states in two different models: present and $R$-matrix model of [7]. The numbers with an asterisk denote prediction from correlation of Figs. 2 and 3 and that with a dagger denotes accurate prediction in Ref. [19].

\begin{tabular}{|c|c|c|c|c|c|c|c|}
\hline & Ref. & 1 state & 3 state & 5 state & 9 state & 22 state & \\
\hline$a^{+}$ & $\begin{array}{c}\text { present } \\
\text { work }\end{array}$ & 4.05 & 3.85 & 3.72 & & & $3.50 *$ \\
\hline$a^{+}$ & [7] & 7.25 & 6.70 & & 5.51 & 5.20 & \\
\hline$r_{0}^{+}$ & $\begin{array}{c}\text { present } \\
\text { work }\end{array}$ & 1.82 & 1.72 & 1.67 & & & $1.65^{*}$ \\
\hline$r_{0}^{+}$ & [7] & 3.07 & 2.98 & & 2.63 & 2.52 & \\
\hline$E_{B}$ & $\begin{array}{c}\text { present } \\
\text { work }\end{array}$ & 0.87 & 0.98 & 1.05 & & & $1.067 \dagger$ \\
\hline$E_{B}$ & [7] & 0.263 & 0.326 & & 0.543 & 0.634 & \\
\hline
\end{tabular}

excitation threshold are shown in Fig. 1. We compare the $\mathrm{S}$-wave phase shifts with the static-exchange phase shift of Hara and Fraser [5] and the three-state close-coupling approximation (CCA) phase shifts of Sinha, Chaudhury, and Ghosh [10]. We also show our static-exchange phase shifts. The phase shifts of Hara and Fraser are identical with the static exchange results of Ref. [10]. The phase shifts of Sinha, Chaudhury, and Ghosh and of Hara and Fraser suggest that the trend of convergence of the $S$-wave phase shifts of Ref. [10] is in the direction of the present phase shifts.

Because of the existence of a low-energy effective-range expansion, the binding energy of a weakly bound singlet Ps-H state should be correlated with the $S$-wave singlet scattering length in different model calculations. This is shown in Fig. 2 where we plot the singlet scattering length versus binding energy for several calculations. The straight-line correlation between these two observables for various model calculations implies that a model that produces the correct energy of the Ps-H bound state should also produce the correct scattering length and good low-energy phase shifts. This correlation explicit in the effective-range expansion is a consequence of the dynamics of the problem. The dominance of the short-range part of the interaction is responsible for the appearance of correlation between low-energy observables in a system [20]. In the trinucleon system in the attractive $S$-wave doublet channel all low-energy observables were found to be correlated with binding energy in different model calculations [20,21], which implies if a model yields the correct result for one of the low-energy observables it should also yield the correct result for the others. Such correlations were used to predict different low-energy trinucleon observables from results of different model calculations. These predictions were later confirmed in other rigorous calculations and experiments [21].

Correlation is also possible among other low-energy $S$-wave singlet Ps-H observables that are not obviously related. For example, we find a correlation between $S$-wave singlet Ps- $\mathrm{H}$ binding energy and effective range, which is shown in Fig. 3. In this figure we plot the effective ranges of Refs. [4,7] and the respective binding energies together with the five-state result. We also observe a correlation between $S$-wave singlet Ps-H binding and resonance energies, which
TABLE II. Low-energy elastic cross sections in units of $\pi a_{0}^{2}$ using different basis sets for different $k$ in au, incident energy $E$ $=6.8 k^{2} \mathrm{eV}$.

\begin{tabular}{lcccccc}
\hline \hline$k$ & 1 state & 2 state & 3 state & 4 state & 5 state & 3-H state \\
\hline 0.0 & 26.39 & 24.78 & 23.35 & 23.35 & 22.36 & 24.10 \\
0.1 & 24.81 & 23.44 & 22.04 & 22.04 & 21.18 & 22.89 \\
0.2 & 21.24 & 20.35 & 19.02 & 19.00 & 18.43 & 19.89 \\
0.3 & 17.60 & 17.13 & 15.83 & 15.83 & 15.51 & 16.79 \\
0.4 & 14.82 & 14.62 & 13.34 & 13.34 & 13.18 & 14.42 \\
0.5 & 12.76 & 12.72 & 11.44 & 11.43 & 11.35 & 12.58 \\
0.6 & 11.01 & 11.05 & 9.72 & 9.72 & 9.68 & 10.89 \\
0.7 & 9.42 & 9.55 & 8.14 & 8.14 & 8.10 & 9.27 \\
0.8 & 8.02 & 7.69 & 6.20 & 6.19 & 6.19 & 7.82 \\
\hline \hline
\end{tabular}

is shown in Fig. 4. The essentially exact resonance and binding energies $[18,19]$ lie on the line in Fig. 4 obtained by our calculation and those of Refs. [4,7]. The reproduction of the correct value of the singlet Ps-H effective range and resonance energy in addition to the scattering length assures proper variation of the phase shift in our model. The previous calculations [4-7] have possibly not converged well as they do not produce the correct energies of Ps-H bound state and resonance. The five-state model reproduces the positions of the Ps-H bound state and resonance fairly accurately, and so it is expected that the present singlet scattering length $3.72 a_{0}$, effective range $1.67 a_{0}$, phase shifts, and low-energy cross sections are closer to the converged results than those of previous calculations. The correlations of Fig. 2 and 3 suggest that the correct singlet scattering length and effective range, corresponding to the accurate Ps-H binding energy of $1.067 \mathrm{eV}$ [19], should be $3.5 a_{0}$ and $1.65 a_{0}$, respectively, in close agreement with our model calculation. In the triplet case there is no bound state and no interesting correlation is observed.

To illustrate the trend of convergence of our calculation, we show in Table I the results for singlet scattering length, effective range, and binding energies for one-, three-, and five-state schemes with model exchange potential and com-

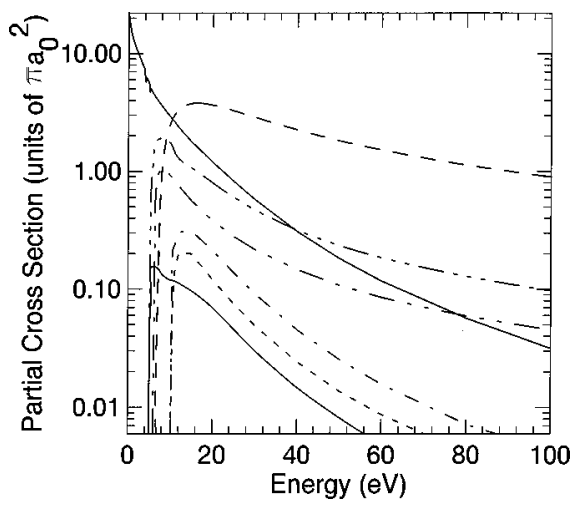

FIG. 5. Partial Ps-H cross sections from the five-state model at different Ps energies: $\mathrm{Ps}(1 s) \mathrm{H}(1 s)$ elastic (upper full line), $\operatorname{Ps}(2 s) \mathrm{H}(1 s)$ excitation (lower full line), $\operatorname{Ps}(1 s) \mathrm{H}(2 s)$ excitation (dotted line), $\operatorname{Ps}(1 s) \mathrm{H}(2 p)$ excitation (dashed-dotted line), $\operatorname{Ps}(2 \mathrm{p}) \mathrm{H}(1 \mathrm{~s})$ excitation (dashed-triple-dotted line), Ps-ionization Born cross section (dashed line), Ps excitation $(n \geqslant 3)$, and Born cross section (dashed-double-dotted line). 
TABLE III. Low-energy phase shifts in radians and ortho-Ps $(1 s)$ to para-Ps $(1 s)$ conversion cross sections in units of $\pi a_{0}^{2}$ for the five-state model for different $k$ in au. The entries for $k=0$ correspond to the scattering lengths in units of $a_{0}$, incident energy $E=6.8 \mathrm{k}^{2} \mathrm{eV}$. The numbers in square brackets denote powers of ten.

\begin{tabular}{lccccccc}
\hline \hline $\begin{array}{l}k \\
(\mathrm{au})\end{array}$ & $\begin{array}{c}\delta_{0}^{+} \\
(\mathrm{rad})\end{array}$ & $\begin{array}{c}\delta_{0}^{-} \\
(\mathrm{rad})\end{array}$ & $\begin{array}{c}\delta_{1}^{+} \\
(\mathrm{rad})\end{array}$ & $\begin{array}{c}\delta_{1}^{-} \\
(\mathrm{rad})\end{array}$ & $\begin{array}{c}\delta_{2}^{+} \\
(\mathrm{rad})\end{array}$ & $\begin{array}{c}\delta_{2}^{-} \\
(\mathrm{rad})\end{array}$ & $\begin{array}{c}\sigma_{\text {quen }} \\
\left(\pi a_{0}^{2}\right)\end{array}$ \\
\hline 0.0 & 3.72 & 1.68 & & & & & 1.02 \\
0.1 & 2.78 & $-1.67[-1]$ & $4.77[-3]$ & $-2.33[-3]$ & $1.8[-5]$ & $-1.4[-5]$ & 0.99 \\
0.2 & 2.44 & $-3.27[-1]$ & $3.70[-2]$ & $-1.67[-2]$ & $5.3[-4]$ & $-4.0[-4]$ & 0.91 \\
0.3 & 2.14 & $-4.74[-1]$ & $1.16[-1]$ & $-4.76[-2]$ & $3.5[-3]$ & $-2.6[-3]$ & 0.93 \\
0.4 & 1.89 & $-6.02[-1]$ & $2.39[-1]$ & $-9.18[-2]$ & $1.2[-2]$ & $-8.6[-3]$ & 1.07 \\
0.5 & 1.68 & $-7.06[-1]$ & $3.72[-1]$ & $-1.42[-1]$ & $2.9[-2]$ & $-2.0[-2]$ & 1.21 \\
0.6 & 1.52 & $-7.84[-1]$ & $4.78[-1]$ & $-1.90[-1]$ & $5.5[-2]$ & $-3.6[-2]$ & 1.21 \\
0.7 & 1.43 & $-8.33[-1]$ & $5.41[-1]$ & $-2.28[-1]$ & $8.8[-2]$ & $-5.5[-2]$ & 1.10 \\
0.8 & 4.12 & $-8.51[-1]$ & $5.69[-1]$ & $-2.47[-1]$ & $1.2[-1]$ & $-7.3[-2]$ & 1.07 \\
\hline \hline
\end{tabular}

pare these with the conventional $R$-matrix calculation of Ref. [7] for different numbers of coupled states. In this table we also show the predictions for the scattering length and effective range obtained from correlations in Figs. 2 and 3 consistent with the correct Ps-H binding [19]. The triplet scattering lengths for the one-, three-, and five-state models, which do not provide any correlation, are $1.83 a_{0}, 1.69 a_{0}$, and $1.68 a_{0}$, respectively.

The model calculation leads to reasonable convergence for cross section and phase shifts at low energies as the number of states is increased. This is illustrated for low-energy cross sections in Table II for different basis sets. Finally, we present the low-energy phase shifts and quenching cross sections of the five-state model in Table III. As in Ref. [7], the quenching cross section has a minimum between 0 and $1 \mathrm{eV}$ and a maximum between 1 and $2 \mathrm{eV}$. However, our lowenergy quenching as well as elastic cross sections are somewhat smaller than those of Ref. [7] and are expected to be more converged.
In Fig. 5 we plot the $\mathrm{Ps}(1 s) \mathrm{H}(1 s), \operatorname{Ps}(2 s) \mathrm{H}(1 s)$, $\operatorname{Ps}(2 p) \mathrm{H}(1 s), \operatorname{Ps}(1 s) \mathrm{H}(2 s)$, and $\mathrm{Ps}(1 s) \mathrm{H}(2 p)$ cross sections for the five-state calculation, and the Born cross sections for $n \geqslant 3$ Ps excitations and Ps ionization. These cross sections are also exhibited in Table IV. The total cross section is plotted in Fig. 6 where we compare our results with those of the 22-pseudo-state $R$ matrix and three-state CCA calculations of Refs. [6,7]. In the absence of experimental results on Ps-H scattering, we compare the total cross section with the total Ps- $\mathrm{H}_{2}$ cross section data $(\mathbf{O})$ reduced by a factor of 2 [2]. This should provide a fair comparison except at very low energies. The experimental trend, which clearly demonstrates a broad maximum in the total cross section for all the Ps-impact scattering problems around $20 \mathrm{eV}$ [1] and possibly a minimum near the $\operatorname{Ps}(2 s)$ excitation threshold [12], is correctly reproduced in our calculation. The Psionization cross section is largely responsible for producing this trend in Ps-H scattering and also in Ps-He and $\mathrm{Ps}-\mathrm{H}_{2}$ scattering $[12,14]$. The 22-pseudo-state calculations of Refs.

TABLE IV. Ps-H partial cross sections in units of $\pi a_{0}^{2}$ at different positronium energies. The numbers in square brackets denote powers of ten.

\begin{tabular}{|c|c|c|c|c|c|c|c|}
\hline $\begin{array}{l}E \\
(\mathrm{eV})\end{array}$ & $\begin{array}{c}\operatorname{Ps}(1 s)- \\
\mathrm{H}(1 s)\end{array}$ & $\begin{array}{c}\operatorname{Ps}(2 s)- \\
\mathrm{H}(1 s)\end{array}$ & $\begin{array}{c}\operatorname{Ps}(2 p)- \\
\mathrm{H}(1 s)\end{array}$ & $\begin{array}{c}\operatorname{Ps}(1 s)- \\
\mathrm{H}(2 s)\end{array}$ & $\begin{array}{c}\operatorname{Ps}(1 s)- \\
\mathrm{H}(2 p)\end{array}$ & $\begin{array}{c}\operatorname{Ps}(n \geqslant 3)- \\
\mathrm{H}(1 s)\end{array}$ & $\begin{array}{l}\text { Ps-ion- } \\
\mathrm{H}(1 s)\end{array}$ \\
\hline 5.08 & 4.70 & & & & & & \\
\hline 5.5 & 4.88 & $1.01[-1]$ & $7.92[-1]$ & & & & \\
\hline 6 & 4.53 & $1.55[-1]$ & 1.40 & & & & \\
\hline 6.8 & 4.10 & $1.52[-1]$ & 1.83 & & & $9.06[-1]$ & \\
\hline 8 & 3.59 & $1.34[-1]$ & 1.93 & & & 1.08 & 1.02 \\
\hline 10 & 2.91 & $1.19[-1]$ & 1.74 & & & $9.16[-1]$ & 2.55 \\
\hline 11 & 2.64 & $1.17[-1]$ & 1.45 & $1.38[-1]$ & $2.33[-1]$ & $8.26[-1]$ & 3.02 \\
\hline 12 & 2.34 & $1.12[-1]$ & 1.28 & $2.06[-1]$ & $3.04[-1]$ & $7.45[-1]$ & 3.35 \\
\hline 15 & 1.78 & $9.64[-2]$ & 1.07 & $1.99[-1]$ & $3.10[-1]$ & $5.60[-1]$ & 3.76 \\
\hline 20 & 1.19 & $6.91[-2]$ & $7.93[-1]$ & $1.20[-1]$ & $2.11[-1]$ & $3.79[-1]$ & 3.64 \\
\hline 25 & $8.17[-1]$ & $4.54[-2]$ & $5.98[-1]$ & $7.48[-2]$ & $1.37[-1]$ & $2.78[-1]$ & 3.26 \\
\hline 30 & $5.73[-1]$ & $3.00[-2]$ & $4.68[-1]$ & $4.88[-2]$ & $9.09[-2]$ & $2.16[-1]$ & 2.87 \\
\hline 40 & $3.08[-1]$ & $1.47[-2]$ & $3.16[-1]$ & $2.42[-2]$ & $4.52[-2]$ & $1.46[-1]$ & 2.25 \\
\hline 60 & $1.17[-1]$ & $5.11[-3]$ & $1.85[-1]$ & $8.71[-3]$ & $1.57[-2]$ & $8.50[-2]$ & 1.52 \\
\hline 80 & $5.65[-2]$ & $2.37[-3]$ & $1.28[-1]$ & $4.08[-3]$ & $7.20[-3]$ & $5.90[-2]$ & 1.13 \\
\hline 100 & $3.15[-2]$ & $1.29[-3]$ & $9.72[-2]$ & $2.22[-3]$ & $3.87[-3]$ & $4.50[-2]$ & 0.90 \\
\hline
\end{tabular}




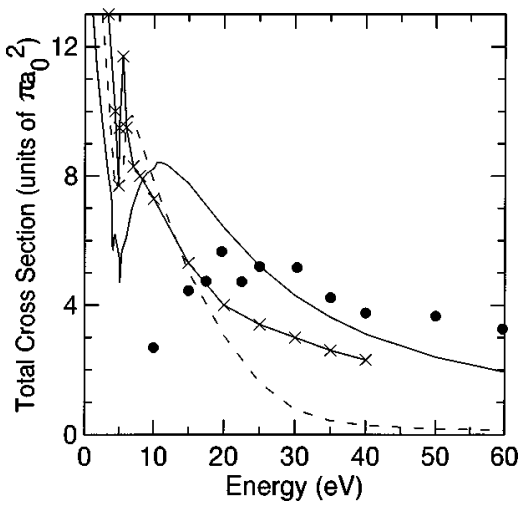

FIG. 6. Total cross section for Ps-H scattering at different Ps energies: present total (full line), three-Ps-state CCA (dotted line, Ref. [6]), target-elastic total of 22-pseudostate model including Ps ionization and excitations (full line with crosses, Ref. [7]), and Ps- $\mathrm{H}_{2}$ experiment reduced by a factor of $2(\boldsymbol{\bullet}$, Refs. [1]).

$[7,8]$ do not have this trend even after including Psionization and $\mathrm{H}$-excitation and ionization cross sections. The Ps-H cross sections of Ref. [6] shown in Fig. 5 do not include higher excitations and ionizations of Ps and $\mathrm{H}$; but the trend of their result suggests that it may agree with the experimental trend of a maximum if these cross sections are included. However, at low energies our cross sections are much smaller than those of Refs. [6,7].

Finally, we compare present elastic cross sections with those of other calculations in Fig. 7. We find that the present cross sections agree reasonably well with the 22-pseudostate calculation of Ref. [7] for energies above $10 \mathrm{eV}$. However, both these works disagree strongly with the three-state CCA calculation of Ref. [6], specially at higher energies. The reason for this disagreement is not clear at present.

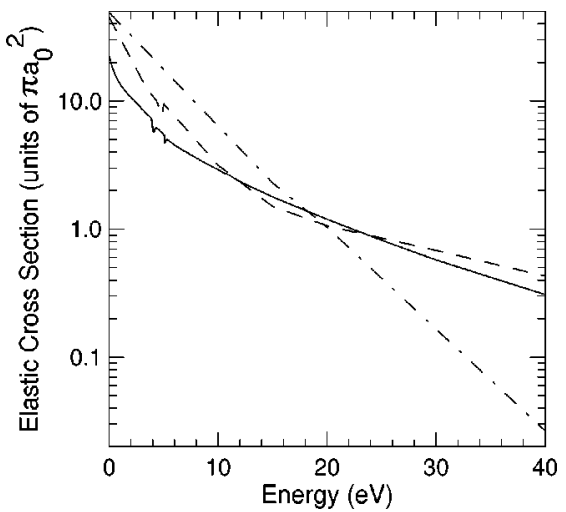

FIG. 7. Elastic cross section at different Ps energies. Present five-state (full line), three-Ps-state CCA (dashed-dotted line, Ref. [6]), and 22-pseudostate model (dashed line, Ref. [7]).
In this study we find that the H-excitation cross sections are much smaller than the Ps-excitation cross sections and the H-excitation channels have less effect on the convergence of the solution at low energies compared to the Psexcitation channels. This is consistent with the fact that the polarizability of the $\mathrm{H}$ atom is one-eighth of that of the Ps atom. In view of this, the difference between our low-energy results and those of Refs. [6,7] seems to be due to their unconverged nature. This is explicit in their estimation for $\mathrm{Ps}-\mathrm{H}$ binding energies. The inclusion of some hydrogen states is not expected to improve their results substantially.

\section{SUMMARY}

We have performed a five-state calculation of Ps-H scattering using a recently proposed nonlocal model exchange potential. The model considers excitation of both Ps and $\mathrm{H}$ atoms and yields cross sections for transitions to following final states starting from the initial state $\operatorname{Ps}(1 s) \mathrm{H}(1 s): \operatorname{Ps}(1 s) \mathrm{H}(1 s), \operatorname{Ps}(2 s) \mathrm{H}(1 s), \operatorname{Ps}(2 p) \mathrm{H}(1 s)$, $\operatorname{Ps}(1 s) \mathrm{H}(2 s)$, and $\operatorname{Ps}(1 s) \mathrm{H}(2 p)$. Higher excitations and ionization of the Ps atom are treated by the Born approximation including exchange. The cross sections are in qualitative agreement with experimental trend. Our five-state model yields singlet and triplet scattering lengths of $3.72 a_{0}$ and $1.68 a_{0}$, and the singlet effective range of $1.67 a_{0}$. The calculation reproduces the singlet $S$-wave Ps-H resonance at $4.01 \mathrm{eV}$ [18] and predicts a Ps-H binding energy of $1.05 \mathrm{eV}$ compared to the accurate binding energy of $1.067 \mathrm{eV}$ [19]. This assures us as to the realistic nature of our model. We observe correlations between the $S$-wave singlet Ps-H binding energy and singlet scattering length, effective range, and resonance energy obtained in different calculations. These correlations of other observables with binding energy demostrate the degree of convergence of various model calculations as can be seen in Figs. 2, 3, and 4. Considering the precise Ps-H binding energy of $1.067 \mathrm{eV}$, correlations in Figs. 2 and 3 suggest a singlet scattering length of $3.5 a_{0}$ and an effective range of $1.65 a_{0}$. The inclusion of higher-order states in our five-state model are not expected to influence the low-energy results significantly as has been demonstrated in Table II, but their effect could be considerable at medium to high energies. A further detailed calculation including these states will help in understanding the dynamics more precisely.

\section{ACKNOWLEDGMENTS}

The work was supported in part by the Conselho Nacional de Desenvolvimento Científico e Tecnológico, Fundação de Amparo à Pesquisa do Estado de São Paulo, and Financiadora de Estudos e Projetos of Brazil.
[1] A. J. Garner, G. Laricchia, and A. Ozen, J. Phys. B 29, 5961 (1996); N. Zafar, G. Laricchia, M. Charlton, and A. Garner, Phys. Rev. Lett. 76, 1595 (1996); A. J. Garner and G. Laricchia, Can. J. Phys. 74, 518 (1996).

[2] M. Skalsey, J. J. Engbrecht, R. K. Bithell, R. S. Vallery, and D. W. Gidley, Phys. Rev. Lett. 80, 3727 (1998).
[3] Y. Nagashima, T. Hyodo, K. Fujiwara, and A. Ichimura, J. Phys. B 31, 329 (1998); K. F. Canter, J. D. McNutt, and L. O. Roellig, Phys. Rev. A 12, 375 (1975).

[4] R. J. Drachman and S. K. Houston, Phys. Rev. A 12, 885 (1975).

[5] S. Hara and P. A. Fraser, J. Phys. B 8, L472 (1975). 
[6] P. K. Sinha, P. Chaudhury, and A. S. Ghosh, J. Phys. B 30, 4643 (1997).

[7] C. P. Campbell, M. T. McAlinden, F. G. R. S. MacDonald, and H. R. J. Walters, Phys. Rev. Lett. 80, 5097 (1998).

[8] M. T. McAlinden, F. G. R. S. MacDonald, and H. R. J. Walters, Can. J. Phys. 74, 434 (1996).

[9] P. K. Biswas and S. K. Adhikari, J. Phys. B 31, 3147 (1998); 31, 5403 (1998). Unfortunately, there was a numerical error in the calculations reported there. The error affected mostly the elastic results below $5 \mathrm{eV}$ and the Ps-ionization cross section.

[10] M. I. Barker and B. H. Bransden, J. Phys. B 1, 1109 (1968); 2, 730 (1969).

[11] P. A. Fraser, J. Phys. B 1, 1006 (1968); N. K. Sarkar and A. S. Ghosh, ibid. 30, 4591 (1997).

[12] P. K. Biswas and S. K. Adhikari, Phys. Rev. A 59, 363 (1999).

[13] M. Comi, G. M. Prosperi, and A. Zecca, Nuovo Cimento 2, 1347 (1983).

[14] P. K. Biswas and S. K. Adhikari, J. Phys. B 31, L737 (1998).

[15] P. K. Biswas and S. K. Adhikari, J. Phys. B 31, L315 (1998).
[16] P. K. Sinha and A. S. Ghosh, Phys. Rev. A 58, 242 (1998).

[17] G. Peach (unpublished) as quoted in Ref. [1].

[18] Y. K. Ho, Phys. Rev. A 17, 1675 (1978).

[19] A. M. Frolov and V. H. Smith, Jr., Phys. Rev. A 55, 2662 (1997); see, also, N. Jiang and D. M. Schrader, Mater. Sci. Forum 255-2, 312 (1997); Y. K. Ho, Phys. Rev. A 34, 609 (1986); A. Ore, Phys. Rev. 83, 665 (1951).

[20] S. K. Adhikari, T. Frederico, and I. D. Goldman, Phys. Rev. Lett. 74, 487 (1995); S. K. Adhikari and K. L. Kowalski, Dynamical Collision Theory and its Applications (Academic Press, San Diego, 1991), Chap. 7.

[21] A. C. Phillips, Nucl. Phys. A 107, 209 (1968); W. Dilg, L. Koester, and W. Nistler, Phys. Lett. 36B, 208 (1971); S. Ishikawa and T. Sasakawa, Phys. Rev. Lett. 56, 317 (1986); B. F. Gibson, Nucl. Phys. A 543, 1c (1992).

[22] This is in fact $-2 \pi^{2}$ times the model exchange potential or the exchange Born term.

[23] D. M. Schrader, F. M. Jacobson, N. P. Frandsen, and U. Mikkelsen, Phys. Rev. Lett. 69, 57 (1992). 